INT-PUB-10-009

\title{
Short-distance and short-time structure of a unitary Fermi gas
}

\author{
Dam T. Son and Ethan G. Thompson \\ Institute for Nuclear Theory, University of Washington, Seattle, WA 98195-1550, USA
}

We consider the operator product expansions for unitarity fermions. We compute the dynamic structure factor $S(\mathbf{q}, \omega)$ at large frequency and wavenumber away from the one-particle peak. The overall normalization of $S(\mathbf{q}, \omega)$ is determined by Tan's contact parameter, and the dependence on $\mathbf{q}$ and $\omega$ is obtained in closed analytic form. We also find energy deposited into the system by small, rapid variations of the inverse scattering length.

PACS numbers: 67.85.Lm

\section{INTRODUCTION}

One of the most interesting systems currently under study is that of fermions with interactions fine-tuned to unitarity [1]. This system is interesting due to the absence of any scale parameter other than the density. The system is strongly interacting and thus presents a challenge for many-body methods.

Recently, these systems have been probed with high-frequency or short-wavelength external probes. By bombarding the fermions with radio-frequency (RF) radiation, which causes a transition from one atomic state to another, one can probe the structure of the unitary Fermi gas. Previous investigations [2-4] have shown that the high frequency tail of the RF line shape is related to the contact parameter, first introduced by Shina Tan $[5,6]$.

In this paper, we address two problems. The first one concerns the dynamic structure factor $S(\mathbf{q}, \omega)$. This factor, in principle, can be measured by Bragg scatterings [7]. At the Fermi momentum and energy scales, the dynamic structure function depends on the complicated many-body physics of the unitary Fermi gas. For much larger $\mathbf{q}$ and $\omega$, the picture becomes simpler. There is a dominant coherent peak in the response function at $\omega=\epsilon_{\mathbf{q}}$. Far away from this peak, the strength of the dynamic structure factor should be proportional to Tan's parameter, the same parameter that characterizes the RF tail. It is easy to understand why this is true: in order to absorb an external "virtual photon" carrying large energy and momentum and far away from the coherent peak, the photon has to hit a particle when it is near a second particle, so that it can give its momentum and energy to both. The absorption rate, therefore, depends on the probability of finding two particles at short distance from each other - which is characterized by Tan's contact parameter.

The problem of the dynamic structure factor is more complicated than that of the RF response. In particular, care should be taken to not violate conservation laws. In this paper we use the operator product expansion (OPE) to facilitate the calculation. The operator product expansion, introduced by Wilson, is a standard method of quantum field theory. 
The use of the OPE for cold atoms was pioneered by Braaten and Platter [8]. The end result for the dynamic structure factor is Eq. (26).

The second problem considered in this paper is the calculation of the energy deposition into a unitary Fermi gas by small, rapid variations of the inverse scattering length. It can be solved using the same OPE methods.

The usefulness of the operator product expansion can be illustrated as follows. Suppose we need to compute the following Green's function

$$
G_{A B}(\omega, \mathbf{q})=\int d t d \mathbf{x} e^{i \omega t-i \mathbf{q} \cdot \mathbf{x}}\langle A(t, \mathbf{x}) B(0, \mathbf{0})\rangle
$$

for large $\omega$ and q. Here "large" means energy and momentum much larger than the typical energy and momentum scales of the state with respect to which the average $\langle\ldots\rangle$ is taken. For the ground state of a unitary Fermi gas, these scales are the Fermi energy and Fermi momentum. Let us also recall that one can associate a local operator $O$ with a scaling dimension $\Delta_{O}$. In our counting scheme, the dimension of momentum is 1 and of energy is 2 (the particle mass $m$ is set to 1 ). Assuming that the operator product expansion exists, one can expand the product $A(t, \mathbf{x}) B(0, \mathbf{0})$ in terms of local operators,

$$
A(t, \mathbf{x}) B(0, \mathbf{0})=\sum_{i}|\mathbf{x}|^{\Delta_{i}-\Delta_{A}-\Delta_{B}} f_{i}\left(\frac{|\mathbf{x}|^{2}}{t}\right) O_{i}(0) .
$$

Here $f_{i}$ are functions of one variable $|\mathbf{x}|^{2} / t$, and $\Delta_{i}$ are the dimensions of $O_{i}$. In contrast to the OPE in relativistically invariant theories, in nonrelativistic theories the OPE coefficients are not constant, but are in general functions of this variable. This identity is to be interpreted as an operator identity; in particular, we can take the expectation value of both sides with respect to any state, including thermodynamic states. Taking the average and performing a Fourier transform, one finds

$$
G_{A B}(\omega, \mathbf{q})=\sum_{i} \frac{1}{\omega^{\left(5+\Delta_{i}-\Delta_{A}-\Delta_{B}\right) / 2}} c_{i}\left(\frac{q^{2}}{\omega}\right)\left\langle O_{i}\right\rangle, \quad q \equiv|\mathbf{q}| .
$$

On the right hand side, the higher the dimension of $O_{i}$, the more rapidly its contribution decays in the limit of large momentum/energy (to be precise, the limit considered in this paper will be $\omega \rightarrow \infty, \mathbf{q} \rightarrow \infty, q^{2} / \omega=$ fixed.) Thus, the leading behavior of the Green function is dominated by those few operators in the OPE with smallest scaling dimension.

The expectation values of the operators $O_{i}$, are, in general, not computable theoretically because they depend on many-body physics. Thus, they should be considered as numbers parameterizing the many-body state. The OPE coefficients $c_{i}$, however, depend only on few-body physics (although, the number of bodies increases with increasing complexity of the operator $O_{i}$ ), and hence can be computed reliably, at least for simple operators $A, B$ and $O_{i}$. In this way, the functional dependence of $G_{A B}$ on frequency and wavenumber can be expressed in terms of a few numbers which have to be determined experimentally or numerically. 
We now discuss the operators of lowest dimension. The unitary fermions are described by the Lagrangian

$$
\mathcal{L}=i \psi^{\dagger} \partial_{t} \psi-\frac{|\nabla \psi|^{2}}{2}+\psi_{2} \psi_{1} \phi^{*}+\psi_{1}^{\dagger} \psi_{2}^{\dagger} \phi-c_{0}^{-1} \phi^{*} \phi .
$$

We set the fermion mass to one. The indices 1,2 refer to the two spin polarizations. If one integrates out $\phi$, the saddle point for this field is at $\phi=c_{0} \psi_{2} \psi_{1}$. This Lagrangian is therefore equivalent to that with a four-Fermi interaction $c_{0} \psi_{1}^{\dagger} \psi_{2}^{\dagger} \psi_{2} \psi_{1}$. We will use dimensional regularization, where setting $c_{0}^{-1}=0$ corresponds to fine-tuning the interaction to infinite scattering length. The propagator of the $\phi$ field is completely determined by its self-energy, and is equal to

$$
D(\omega, \mathbf{q})=-\frac{4 \pi}{\sqrt{q^{2} / 4-\omega-i 0}} .
$$

Let us discuss the operators which may have nonzero expectation value in a unitary Fermi gas. For our applications, we will need to consider only operators which do not carry particle numbers. Moreover, we assume the ground state to be isotropic, so we need to look only at operators with vanishing orbital angular momentum. The following two operators have the lowest dimensions: $n_{i}=\psi_{i}^{\dagger} \psi_{i}(i=1,2)$ and $\phi^{*} \phi$. The operators $n_{i}$ are the particle number densities and have dimension 3. The operator $\phi^{*} \phi$ has dimension equal to 4 . This can be seen by computing the dimension of $\phi$ from Eq. (5). An alternative way to find the dimension of $\phi$ is to use the operator-state correspondence, according to which the dimension of $\phi$ is the ground state energy of a system of one spin-up and one spin-down particle in a harmonic trap with unit oscillator frequency. This ground state energy is 2 .

As we shall see, the expectation value $\left\langle\phi^{*} \phi\right\rangle$ can be identified with Tan's contact parameter. Together, these two operators dominate the high-momentum behavior of correlation functions, including the dynamic structure factor.

\section{SINGLE-PARTICLE GREEN'S FUNCTION}

To establish the relationship between $\left\langle\phi^{*} \phi\right\rangle$ and Tan's contact parameter, let us apply the method of the OPE to the one-particle Green's function

$$
i G(\omega, \mathbf{q})=\int d t d \mathbf{x} e^{i \omega t-i \mathbf{q} \cdot \mathbf{x}}\left\langle T \psi_{1}(t, \mathbf{x}) \psi_{1}^{\dagger}(0, \mathbf{0})\right\rangle,
$$

where $T$ denotes time ordering. ${ }^{1}$ At the end we will be interested only in the case of $t<0$, where the Green's function is $-\left\langle\psi^{\dagger}(0) \psi(t, \mathbf{x})\right\rangle$, but the Feynman diagrams are readily available for the time-ordered Green's function.

According to the previous discussion, we can write

$$
G(\omega, \mathbf{q})=C_{n}(\omega, \mathbf{q})\left\langle\psi_{2}^{\dagger} \psi_{2}\right\rangle+C_{\phi^{*} \phi}(\omega, \mathbf{q})\left\langle\phi^{*} \phi\right\rangle+\cdots
$$

${ }^{1}$ Y. Nishida [9] has essentially arrived at a similar derivation. 
(We shall see why there is no $\left\langle\psi_{1}^{\dagger} \psi_{1}\right\rangle$ term below.)

To compute $C_{n}$, we use the Feynman diagram in Fig. 1.



FIG. 1: The diagram that gives $C_{n}$ in Eq. (7). The large momentum and energy flow along lines carrying label "q."

Physically, this diagram describes the interaction of a particle carrying large momentum and frequency $(\omega, \mathbf{q})$ with particles already in the medium. The energy and momentum of the particles in the medium can be neglected compared to $(\omega, \mathbf{q})$. The "hard" particle interacts with particles of type 2 in the medium with the result,

$$
C_{n}(\omega, \mathbf{q})=-\frac{4 \pi}{\left(\omega-q^{2} / 2+i 0\right)^{2} \sqrt{q^{2} / 4-\omega-i 0}} .
$$

One can see immediately that $C_{n}(\omega, \mathbf{q})$ has singularities only in the lower-half plane, and thus does not contribute to the Green's function for $t<0$. This can already be seen from the Feynman diagram in Fig. 1: one can go directly from the initial point to the final point, following the direction of the propagators (fermions and scalars). Recall that the propagators are all retarded.

Consider now the OPE coefficient of $C_{\phi^{*} \phi}$. It can be found by computing the fermion propagator in the background field of $\phi$. One diagram that contributes to $C_{\phi^{*} \phi}$ is as in Fig. 2. The dashed lines carry zero energy and momentum, and go to the "condensate"

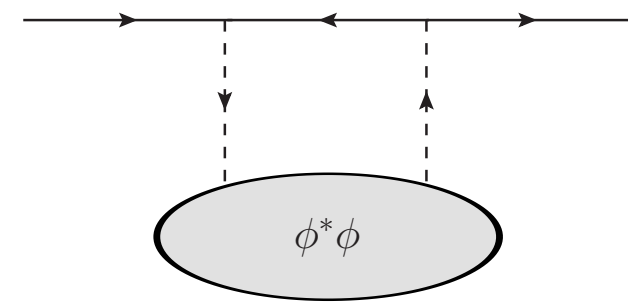

FIG. 2: A contribution of $\left\langle\phi^{*} \phi\right\rangle$ to the fermion propagator

$\left\langle\phi^{*} \phi\right\rangle$. Fig. 2 is not the sole diagram contributing to $C_{\phi^{*} \phi}$; there is an infinite number of diagrams contributing to this coefficient, like those depicted in Fig. 3 and obvious subsequent iterations. It can be seen that, in all diagrams of the type of Fig. 3, one can go from one end of the diagram to the other by following the arrows, which is not true in the case of Fig. 2. 



FIG. 3: The other diagrams contributing to the fermion propagator

Thus, if our ultimate goal is to compute the Green's function for $t<0$, it is sufficient to just evaluate the diagram in Fig. 2.

From the Feynman rules, the contribution of Fig. 2 to $C_{\phi^{*} \phi}$ is

$$
-\frac{1}{\left(\omega-\epsilon_{\mathbf{q}}+i 0\right)^{2}\left(-\omega-\epsilon_{\mathbf{q}}+i 0\right)},
$$

where $\epsilon_{\mathbf{q}}=q^{2} / 2$.

With this information we can find the leading nontrivial contribution to the Green's function for $t<0$ :

$$
\begin{aligned}
\int d \mathbf{x} e^{-i \mathbf{q} \cdot \mathbf{x}}\left\langle\psi_{1}^{\dagger}(0, \mathbf{0}) \psi_{1}(t, \mathbf{x})\right\rangle=-i \int \frac{d \omega}{2 \pi} e^{-i \omega t} C_{\phi^{*} \phi}(\omega, \mathbf{q})\left\langle\phi^{*} \phi\right\rangle & \\
& =\frac{1}{q^{4}} \exp \left(i \frac{q^{2}}{2} t\right)\left\langle\phi^{*} \phi\right\rangle+\cdots, \quad t<0 .
\end{aligned}
$$

In the limit $t \rightarrow-0$, we find the tail of the distribution function to be

$$
n_{\mathbf{q}}=\frac{\left\langle\phi^{*} \phi\right\rangle}{q^{4}},
$$

which allows us to establish $\left\langle\phi^{*} \phi\right\rangle=C$, where $C$ is Tan's contact parameter [5, 6].

One can also show that the diagrams in Fig. 3 do not contribute to the imaginary part of $G(\omega, \mathbf{q})$ for $\omega<0$. The latter receives only a contribution from Fig. 2, which is

$$
\omega<0: \quad \operatorname{Im} G(\omega, \mathbf{q})=\frac{\left\langle\phi^{*} \phi\right\rangle}{q^{4}} \pi \delta\left(\omega+\epsilon_{\mathbf{q}}\right) .
$$

The peak at $\omega=-\epsilon_{\mathbf{q}}$ is already discussed in Refs. [11, 12]. At this level, we are not able to resolve the structure of the peak.

\section{RF SPECTROSCOPY}

Consider now a system where, in addition to the 'up' and 'down' fermions included in the earlier Lagrangian, a third species of fermion is added which does not interact with the other two fermions. Suppose we turn on a photon field which converts atoms of type one into atoms of type three. The absorption rate is proportional to the imaginary part of the Green's function of

$$
O_{13}=\psi_{3}^{\dagger} \psi_{1}
$$


We thus need to compute the OPE expansion of

$$
\Pi(\omega, \mathbf{q})=-i \int d t d \mathbf{x} e^{i \omega t-i \mathbf{q} \cdot \mathbf{x}}\left\langle T O_{13}(t, \mathbf{x}) O_{13}^{\dagger}(0, \mathbf{0})\right\rangle=C_{n}\left\langle n_{1}\right\rangle+C_{\phi^{*} \phi}\left\langle\phi^{*} \phi\right\rangle+\cdots
$$

The coefficient $C_{n}$ is obtained from the diagram of Fig. 4,

$$
C_{n}=\frac{1}{\omega-\epsilon_{\mathbf{q}}+i 0} .
$$

The external wavy lines in this diagram represent the operator $O_{13}$. The interpretation of this formula is obvious. The dominant part of the response function is the same as a collection of noninteracting particles with zero momentum, and has a peak at $\omega=\epsilon_{\mathbf{q}}$.

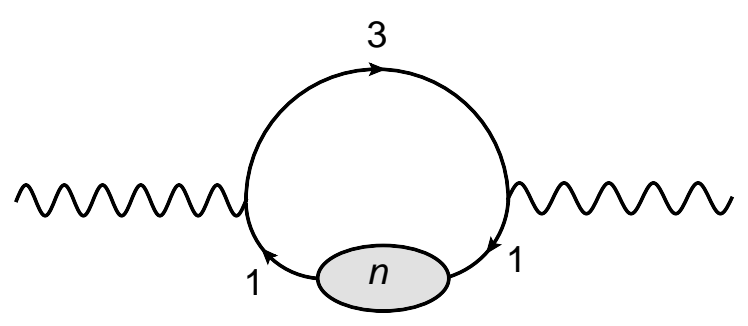

FIG. 4: Diagram contributing to $C_{n}$

Let us now compute the diagram which determines the coefficient of $\phi^{*} \phi$ in the OPE (Fig. 5). We find

$$
\begin{aligned}
\Pi(\omega, \mathbf{q})= & \cdots-i \int \frac{d p_{0} d \mathbf{p}}{(2 \pi)^{4}} \frac{1}{\left(p_{0}-\epsilon_{\mathbf{p}}+i 0\right)^{2}} \frac{1}{p_{0}+\omega-\epsilon_{\mathbf{p}+\mathbf{q}}} \frac{1}{p_{0}+\epsilon_{\mathbf{p}}-i 0}\left\langle\phi^{*} \phi\right\rangle \\
\quad+(\omega \rightarrow-\omega, \mathbf{q} \rightarrow-\mathbf{q})+\cdots & \\
= & \cdots+\int \frac{d \mathbf{p}}{(2 \pi)^{3}} \frac{1}{4 \epsilon_{\mathbf{p}}^{2}} \frac{1}{\omega-\epsilon_{p}-\epsilon_{\mathbf{p}+\mathbf{q}}+i 0}\left\langle\phi^{*} \phi\right\rangle+(\omega \rightarrow-\omega, \mathbf{q} \rightarrow-\mathbf{q})+\cdots
\end{aligned}
$$

This integral is divergent in the infrared. We can isolate the divergent part of the integral as

$$
\frac{\left\langle\phi^{*} \phi\right\rangle}{\omega-\epsilon_{\mathbf{q}}+i 0} \int \frac{d \mathbf{p}}{(2 \pi)^{3}} \frac{1}{4 \epsilon_{\mathbf{p}}^{2}}+\int \frac{d \mathbf{p}}{(2 \pi)^{3}} \frac{1}{4 \epsilon_{\mathbf{p}}^{2}}\left(\frac{1}{\omega-\epsilon_{\mathbf{p}}-\epsilon_{\mathbf{p}+\mathbf{q}}+i 0}-\frac{1}{\omega-\epsilon_{\mathbf{q}}+i 0}\right)\left\langle\phi^{*} \phi\right\rangle .
$$

It is clear that the first (divergent) part is actually included in the first term in the OPE (15), i.e., it represents the contribution of the condensate $\left\langle\phi^{*} \phi\right\rangle$ to the number density $n$. Subtracting this contribution, the second part gives $C_{\phi^{*} \phi}$,

$$
C_{\phi^{*} \phi}=\int \frac{d \mathbf{p}}{(2 \pi)^{3}} \frac{1}{4 \epsilon_{p}^{2}}\left(\frac{1}{\omega-\epsilon_{p}-\epsilon_{\mathbf{p}+\mathbf{q}}+i 0}-\frac{1}{\omega-\epsilon_{\mathbf{q}}+i 0}\right)+(\omega \rightarrow-\omega, \mathbf{q} \rightarrow-\mathbf{q}) .
$$

We now restrict ourselves to the imaginary part at $\mathbf{q}=0$. We have

$$
I(\omega)=-\frac{1}{\pi} \operatorname{Im} \Pi(\omega, \mathbf{0})=\frac{\left\langle\phi^{*} \phi\right\rangle}{4 \pi^{2}} \frac{1}{\omega^{3 / 2}},
$$

which coincides with the result obtained previously by other authors $[3,4]$. 


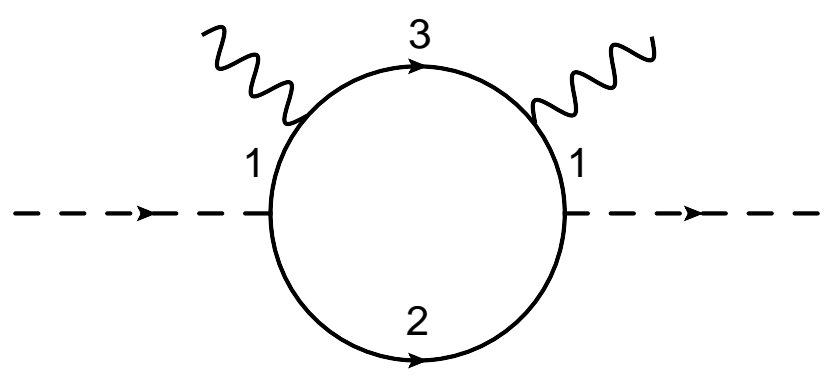

FIG. 5: Diagram contributing to $C_{\phi^{*} \phi}$. The external dashed lines, which carry zero energy and momentum, go to the "condensate" $\left\langle\phi^{*} \phi\right\rangle$ which is not depicted for simplicity.

\section{DYNAMIC STRUCTURE FACTOR}

Having tested the OPE method on the simple examples above, we will now discuss the dynamic structure factor. The dynamic structure factor can be defined as the imaginary part of the Green function of the density,

$$
G_{n n}(\omega, \mathbf{q})=-i \int d t d \mathbf{q} e^{i \omega t-i \mathbf{k} \cdot \mathbf{x}}\langle\operatorname{Tn}(t, \mathbf{x}) n(0, \mathbf{0})\rangle=C_{n}\langle n\rangle+C_{\phi^{*} \phi}\left\langle\phi^{*} \phi\right\rangle+\cdots
$$

Namely,

$$
S(\mathbf{q}, \omega)=-\frac{1}{\pi} \operatorname{Im} G_{n n}(\omega, \mathbf{q}), \quad \omega>0 .
$$

The coefficient $C_{n}$ is

$$
C_{n}=G(\omega, \mathbf{q})+G(-\omega,-\mathbf{q})=\frac{2 \epsilon_{\mathbf{q}}}{\omega^{2}-\epsilon_{\mathbf{q}}^{2}+i 0},
$$

and corresponds to the one-particle peak $\sim \delta\left(\omega-\epsilon_{\mathbf{q}}\right)$ in the dynamic structure factor. This peak dominates all sum rules. However, we are interested in the structure factor far away from this peak, hence we need to compute the OPE coefficient of the next operator, $\phi^{*} \phi$.

The diagrams contributing to $C_{\phi^{*} \phi}$ are sketched schematically in Fig. 6.

The contribution from the first diagram is exactly twice the diagram in the RF case,

$$
C_{\phi^{*} \phi}^{(1)}=2 \int \frac{d \mathbf{p}}{(2 \pi)^{3}} \frac{1}{4 \epsilon_{\mathbf{p}}^{2}}\left(\frac{1}{\omega-\epsilon_{\mathbf{p}}-\epsilon_{\mathbf{p}+\mathbf{q}}+i 0}-\frac{1}{\omega-\epsilon_{\mathbf{q}}+i 0}\right)+(\omega \rightarrow-\omega, \mathbf{q} \rightarrow-\mathbf{q}) .
$$

The second diagram gives

$$
C_{\phi^{*} \phi}^{(2)}=2 \int \frac{d \mathbf{p}}{(2 \pi)^{3}} \frac{1}{4 \epsilon_{\mathbf{p}} \epsilon_{\mathbf{p}+\mathbf{q}}}\left(\frac{1}{\omega-\epsilon_{\mathbf{p}}-\epsilon_{\mathbf{p}+\mathbf{q}}+i 0}-\frac{1}{\omega+\epsilon_{\mathbf{p}}+\epsilon_{\mathbf{p}+\mathbf{q}}-i 0}\right) .
$$

The third diagram is the product of three pieces, each of which can be computed separately. At the end one finds

$$
C_{\phi^{*} \phi}^{(3)}=\left[-2 \int \frac{d \mathbf{p}}{(2 \pi)^{3}} \frac{1}{2 \epsilon_{\mathbf{p}}} \frac{1}{\omega-\epsilon_{\mathbf{p}}-\epsilon_{\mathbf{p}+\mathbf{q}}+i 0}\right]^{2} \frac{-4 \pi}{\sqrt{\frac{1}{2} \epsilon_{\mathbf{q}}-\omega-i 0}}+(\omega \rightarrow-\omega, \mathbf{q} \rightarrow-\mathbf{q}) .
$$



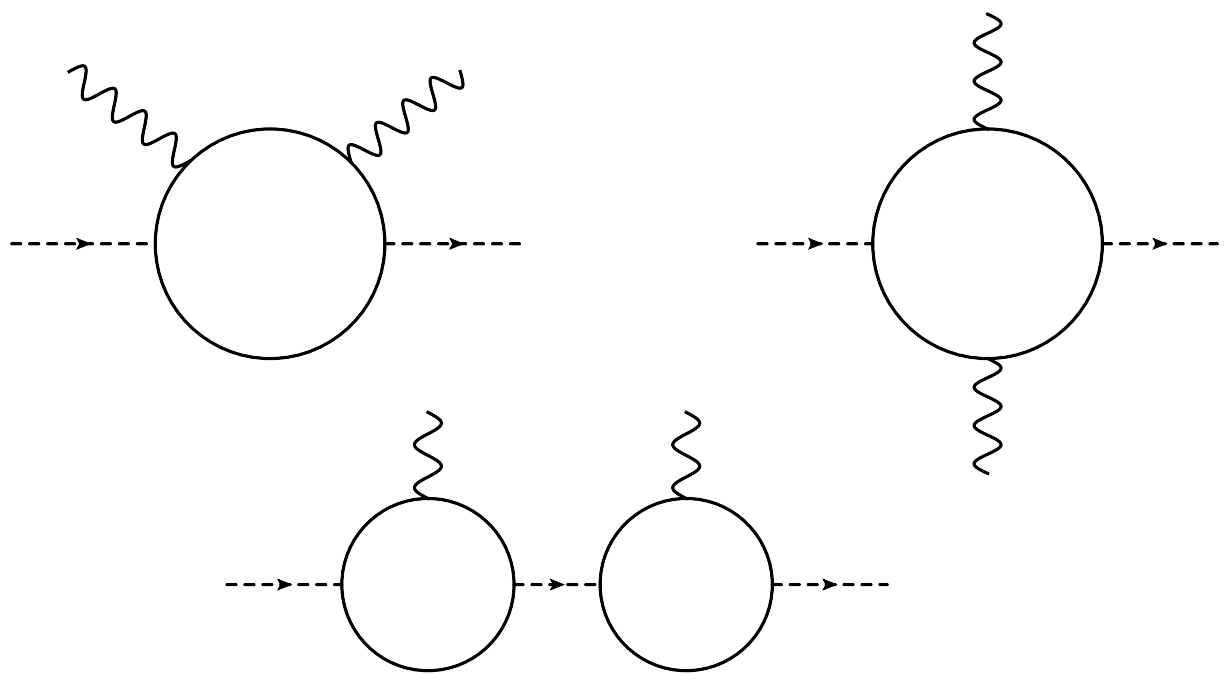

FIG. 6: Schematic diagrams contributing to $C_{\phi \phi}$. Each diagram represents a number of Feynman graphs where the photon lines are attached to different fermion lines

Evaluating the imaginary part of the correlation function for positive $\omega$, we find that it is zero when $\omega<q^{2} / 4$. This should be the case since $\omega=q^{2} / 4$ is the threshold for knocking out two particles from the medium (recall that all the energy scales associated with the medium, like the chemical potential, are negligible). For $\omega>q^{2} / 4$, the dynamic structure factor is

$$
\begin{aligned}
\frac{S(\mathbf{q}, \omega)}{\left\langle\phi^{*} \phi\right\rangle}=-\frac{1}{\pi} \operatorname{Im} C_{\phi^{*} \phi} & =\frac{1}{2 \pi^{2}} \frac{\sqrt{\omega-q^{2} / 4}}{\left(\omega-q^{2} / 2\right)^{2}}+\frac{1}{2 \pi^{2} \omega q} \ln \frac{\omega+q \sqrt{\omega-q^{2} / 4}}{\left|\omega-q^{2} / 2\right|} \\
& -\frac{1}{\pi^{2} q^{2} \sqrt{\omega-q^{2} / 4}}\left(\ln ^{2} \frac{\omega+q \sqrt{\omega-q^{2} / 4}}{\left|\omega-q^{2} / 2\right|}-\pi^{2} \theta\left(q^{2} / 2-\omega\right)\right) .
\end{aligned}
$$

In Fig. 7 we plot the function $\omega^{3 / 2} S(\omega, \mathbf{q}) /\left\langle\phi^{*} \phi\right\rangle$ as a function of the "Bjorken $x$ " variable, $x=q^{2} / 2 \omega$. This can be thought of as a plot of $S$ at fixed $\omega$ as a function of $q^{2}$. In Fig. 8,

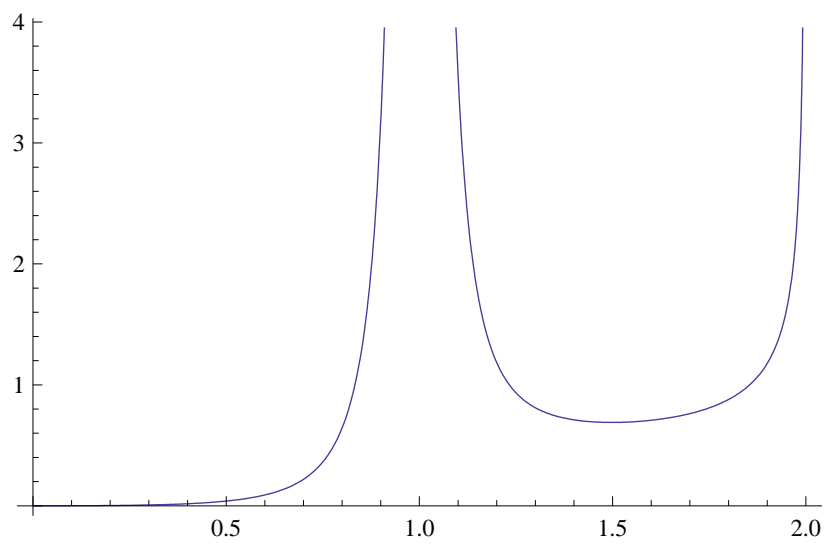

FIG. 7: A plot of $\omega^{3 / 2} S(\mathbf{q}, \omega) /\left\langle\phi^{*} \phi\right\rangle$ as a function of $x=q^{2} / 2 \omega$. 
we plot the same quantity as a function of $x^{-1}=2 \omega / q^{2}$. This plot is basically a plot of $S$ as a function of $\omega$ at fixed $q$.

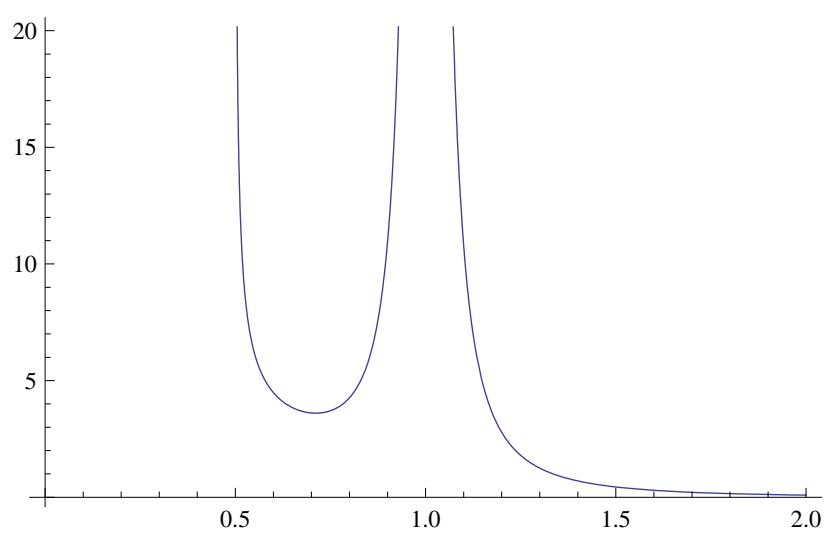

FIG. 8: A plot of $q^{3 / 2} S(\mathbf{q}, \omega) /\left\langle\phi^{*} \phi\right\rangle$ as a function of $x^{-1}=2 \omega / q^{2}$.

As we see from the plots, the dynamic structure factor is positive for all $\omega$ and $\mathbf{q}$, as it should be. It vanishes in the zero momentum, finite frequency limit $\mathbf{q}=0$, as it should due to the conservation of particle number. At small $q^{2} / \omega$, the behavior of the dynamic structure factor is

$$
S(\mathbf{q}, \omega)=\frac{4}{45 \pi^{2}} \frac{q^{4}}{\omega^{7 / 2}}\left\langle\phi^{*} \phi\right\rangle .
$$

The dynamic structure function has a singularity near $\omega=q^{2} / 2$, which is the single-particle peak. It would be incorrect to take the limit $x=q^{2} / 2 \omega \rightarrow 1$ in our formula at fixed $\mathbf{q}$ or fixed $\omega$. Our result is strictly valid in the regime of $\omega \rightarrow \infty, \mathbf{q} \rightarrow \infty$, fixed $x=q^{2} / 2 \omega \neq 1$. The weaker singularity at $\omega=q^{2} / 4(x=2)$ is associated with the fact that near this point, the two particles that are kicked out have small relative momentum, and thus interact strongly with each other.

\section{RESPONSE OF UNITARY GAS TO RAPID CHANGES OF THE INVERSE SCATTERING LENGTH}

We consider in this section the following problem. Assume we make the inverse scattering length $\alpha \equiv a^{-1}$ exhibit periodic behavior in time,

$$
\alpha(t)=a^{-1}(t)=\alpha_{0} \cos \omega t,
$$

and assume the amplitude $\alpha_{0}$ to be small, and the frequency of the oscillations to be large compared to the Fermi energy, $\omega \gg \epsilon_{\mathrm{F}}$. The question is: at what rate is energy deposited into the system?

We know that the inverse scattering length is coupled to the operator $\phi^{*} \phi$, i.e., introducing a finite inverse scattering length corresponds to adding into the Lagrangian a term

$$
\mathcal{L} \rightarrow \mathcal{L}+\frac{\alpha}{4 \pi} \phi^{*} \phi .
$$


The energy deposited into the system, in unit volume and in unit time, can be computed from the formula

$$
\frac{d \epsilon}{d t}=\frac{\alpha_{0}^{2}}{2(4 \pi)^{2}} \omega\left|\operatorname{Im} G_{O O}(\omega, \mathbf{0})\right|,
$$

where $G_{O O}$ is the Greens function of the the operator $O=\phi^{*} \phi$. The OPE of two $O$ operators is easy to compute,

$$
-i\left\langle T \phi^{*} \phi(t, \mathbf{x}) \phi^{*} \phi(0)\right\rangle=\left\langle\phi^{*} \phi\right\rangle[D(t, \mathbf{x})+D(-t,-\mathbf{x})]+\cdots,
$$

where $D(t, \mathbf{x})$ is the scalar propagator [Eq. (5)]. From this we find $\operatorname{Im} G_{O O}(\omega, \mathbf{0})=$ $-4 \pi \omega^{-1 / 2}\left\langle\phi^{*} \phi\right\rangle$. Thus we find the rate of energy deposition,

$$
\frac{d \epsilon}{d t}=\frac{\alpha_{0}^{2}}{8 \pi}\left\langle\phi^{*} \phi\right\rangle \sqrt{\omega} .
$$

If $\alpha(t)$ has the form of a sudden pulse, i.e., is nonzero only in a finite time interval, the total energy deposited into the system, per unit volume, will be related to the Fourier transform $\tilde{\alpha}(\omega)=\int d t e^{i \omega t} \alpha(t)$ by

$$
\epsilon=\frac{\left\langle\phi^{*} \phi\right\rangle}{2 \pi} \int_{0}^{\infty} \frac{d \omega}{2 \pi} \sqrt{\omega}|\tilde{\alpha}(\omega)|^{2} .
$$

\section{CONCLUSION}

In this paper we have shown that the structure of a unitary Fermi gas, at short distance and length scales, can be obtained from the operator product expansion. We computed the OPE of the density operator at two points and derived an expression for the dynamic structure factor. The same technique is applied to the problem of finding the energy deposited into a unitary Fermi gas by a rapid, small oscillation of the scattering length.

As mentioned above, the two-body contribution to $S(\mathbf{q}, \omega)$ vanishes for $\omega<q^{2} / 4$ : this is the two-body threshold. If $\omega>q^{2} / 6$, it is possible to transfer the energy and momentum to three particles instead. In general, when $n-1<x<n$, where $x$ is the "Bjorken $x$ " variable, the dominant contribution to the dynamic structure factor $S(\omega, \mathbf{q})$ in the large $\omega$, fixed $x$ regime is due to the lowest dimensional operator in the OPE that contains $n$ creation and $n$ annihilation operators. The dimension of this operator is $2 \Delta_{n}$, where $\Delta_{n}$ is the ground state energy of a system of $n$ particles in a harmonic potential. Thus we find

$$
S(\mathbf{q}, \omega) \sim\left\langle O_{n}^{\dagger} O_{n}\right\rangle \frac{f_{n}(x)}{\omega^{\Delta_{n}-1 / 2}}
$$

in the limit $\omega \rightarrow \infty, x=$ fixed, and $n-1<x<n(n \geq 3)$. For example, the $n=3$ body contribution to the dynamic structure factor is down by $\omega^{-3.77272}$ since $\Delta_{3} \approx 4.27272$. The larger $n$ is, the faster the contribution of $n$-body physics decreases with increasing momentum. 
The operator product expansion cleanly separates "hard" (large-momentum and/or largeenergy) physics from soft physics. The computation of the OPE coefficients involves computing diagrams in vacuum, with a finite number of particles in the intermediate state. Thus the OPE is a natural way of separating few-body from many-body physics. It application to cold atom physics should be further investigated.

\section{Acknowledgments}

The authors thank G. Baym, E. Braaten, A. L. Fitzpatrick, E. Katz, Y. Nishida, L. Platter, S. Shenker, and S. Tan for discussions. This work is supported, in part, by DOE grant No. DE-FG02-00ER41132 and by University of Washington Royalty Research Fund grant No. 65-8195. After this work was finished, we learned about Ref. [13] which has some overlap with our calculation of the RF response. We also learned from M. Randeria and E. Taylor that they considered $S(\mathbf{q}, \omega)$ in the large- $\omega$, small- $q$ regime and obtained the same $\left\langle\phi^{*} \phi\right\rangle q^{4} / \omega^{7 / 2}$ behavior as in Eq. (27), but with a different numerical coefficient [14].

[1] A. Leggett, Quantum Liquids, Oxford University Press, Oxford, 2006; section 8.4.

[2] P. Pieri, A. Perali, and G. C. Strinati, Nature Phys. 5, 736 (2009) [arXiv:0811.0770].

[3] W. Schneider, V. B. Shenoy, and M. Randeria, arXiv:0903.3006.

[4] R. Haussmann, M. Punk, and W. Zwerger, Phys. Rev. A 80, 063612 (2009) [arXiv:0904.1333].

[5] S. Tan, Ann. Phys. (N.Y.) 323, 2952 (2008) [cond-mat/0505200].

[6] S. Tan, Ann. Phys. (N.Y.) 323, 2971 (2008) [cond-mat/0508320].

[7] H. Hu, E. D. Kuhnle, X.-J. Liu, P. Dyke, M. Mark, P. D. Drummond, P. Hannaford, and C. J. Vale, arXiv:1001.3200.

[8] E. Braaten and L. Platter, Phys. Rev. Lett. 100, 205301 (2008) [arXiv:0803.1125].

[9] Y. Nishida, unpublished notes.

[10] Y. Nishida and D. T. Son, Phys. Rev. D 76, 086004 (2007) [arXiv:0706.3746].

[11] R. Combescot, F. Alzetto, and X. Leyronas, Phys. Rev. A 79, 053640 (2009) [arXiv:0901.4303].

[12] W. Schneider and M. Randeria, arXiv:0910.2693.

[13] E. Braaten, D. Kang, and L. Platter, arXiv:1001.4518.

[14] E. Taylor and M. Randeria, arXiv:1002.0869. 